INPLASY

PROTOCOL

To cite: Shida et al. The Effects of Exergames on Muscle Architecture: A systematic review and meta-analysis protocol. Inplasy protocol 202140054. doi:

10.37766/inplasy2021.4.0054

Received: 09 April 2021

Published: 09 April 2021

Corresponding author:

Nami Shida

shida@tmu.ac.jp

Author Affiliation:

Tokyo Metropolitan University

Support: None.

Review Stage at time of this submission: Preliminary searches.

Conflicts of interest: None declared.

\section{The Effects of Exergames on Muscle Architecture: A systematic review and meta-analysis protocol}

Shida, N1; Yagiz, G2; Yamada, T33.

Review question / Objective: Do exergames improve the architectural parameters of the skeletal muscles?

Condition being studied: Exergame induced architectural alterations in skeletal muscle architecture in human.

Eligibility criteria: Eligibility criteria will be considered as 1) Investigating effects of an exergame intervention; 2) presenting magnetic resonance imaging (MRI) or ultrasound measured alterations in one or more specifically defined muscle architectural parameter(s) as an outcome; 3) being a full-text journal article; 4) being written in the English language.

INPLASY registration number: This protocol was registered with the International Platform of Registered Systematic Review and Meta-Analysis Protocols (INPLASY) on 09 April 2021 and was last updated on 09 April 2021 (registration number INPLASY202140054).

\section{INTRODUCTION}

Review question / Objective: Do exergames improve the architectural parameters of the skeletal muscles?

Rationale: Muscle architecture is a wide term comprising fascicle geometry (fascicle length and pennation angle) and muscle size (anatomical and physiological cross-sectional areas, muscle thickness and muscle length). Larger muscle sizes are strongly associated with higher muscle strength. Additionally, longer fascicle lengths and larger muscle sizes are significantly related to an increased rate of force development, increased power generation, sprint and walking 
performances. Further, smaller muscle sizes and shorter fascicle lengths were found in association with sport and orthopaedic injuries. Due to ageing, significant adverse alterations happen in the muscle size, fascicle length and pennation angle. The decrements in muscle size and muscle functionality have been defined as sarcopenia, which is significantly associated with increased mortality and morbidity in elderly or clinical people. Similarly, decrements in the muscle size were also detected due to the disuse of muscles after bed rest or exposure to microgravity. These muscle atrophies might lead to lesser contractile performance and metabolic dysregulations. Review studies stated that distinct conventional training modalities, i.e. concentric, eccentric, isometric exercises, were found effective to improve distinct muscle architectural parameters. On the other hand, virtual games (exergames) emerged as an alternative tool to enhance physical activity and exercising by the development of technology. Due to its motivating features, exergames increased commitment to exercise. With the recent developments, commercial exergames became capable of imitating resistance training as an addition to previous exergame features such as cycling, dancing, running, walking and playing a sport modality. Despite the crucial importance of the muscle architectural parameters, there is no systematic review investigating the effects of exergames on the skeletal muscle architectural parameters that exists in the literature.

Condition being studied: Exergame induced architectural alterations in skeletal muscle architecture in human.

\section{METHODS}

Search strategy: Databases will be searched by using a combination of the following key terms: ("Active video gaming" OR Avatar OR AVG OR "Commercial game" OR "Computer-based" OR "Computer based" OR "Computer feedback" OR "Computer game" OR "Dance dance revolution" OR "Digital game" OR "Digital- game" OR Exergam* OR "Game training" OR "Game exercise" OR "Game-based" OR "health game" OR IREX OR Kinect OR "Lower limb power rehabilitation" OR Nintendo OR "Play station" OR "Playstation" OR SensBalance OR "Serious game" OR "Serious gaming" OR "Sony EyeToy" OR "Video game" OR "Videogame" OR "Videogame" OR "Video gaming" OR "Video based" OR videobased OR "Video-based" OR "Virtual reality" OR VRT OR Wii OR Wobble OR Xavi* OR XBOX OR X-box) AND ("Muscle architecture" OR "Cross sectional area" OR "Cross-sectional area" OR Fascic* OR "Fiber length" OR "Fibre length" OR "Muscle length" OR "Muscle structure" OR "Muscle thickness" OR "Muscle volume" OR Pennat* OR Pinnat*). In addition to this, reference lists of eligible studies will be screened. Duplicate records will be removed by using the EndNote X9 computer program. After removal of duplicates, remaining records will be independently screened by the first and second authors via using Rayyan- a web and mobile app for systematic reviews. Once eligible studies detected, the reference lists of the studies will be independently screened by the first and second authors. Similarly, data extraction of the eligible studies will be independently done by the lead and second authors. Disagreements about selecting the studies and extracting data will be solved by discussion between the lead and second authors. If the first and second authors cannot decrypt the disagreements, the last author will be consulted. If full-text of articles is not available, university libraries and corresponding authors of the studies will be contacted. In case of missing outcome data, corresponding authors of the studies will be contacted via emails. Preferred Reporting Items for Systematic Reviews and Meta-analyses (PRISMA) statement will be used as the guideline of this review.

Participant or population: Human regardless of age, gender or clinical condition.

Intervention: Exergame interventions. 
Comparator: A non-training control group or a control group that received placebo or usual care interventions will be considered a comparator.

Study designs to be included: Nonrandomised and randomised trials.

Eligibility criteria: Eligibility criteria will be considered as 1) Investigating effects of an exergame intervention; 2) presenting magnetic resonance imaging (MRI) or ultrasound measured alterations in one or more specifically defined muscle architectural parameter(s) as an outcome; 3) being a full-text journal article; 4) being written in the English language.

Information sources: Cochrane Central Register of Controlled Trials (CENTRAL), CINAHL, ProQuest, PubMed, OpenGrey and reference lists of eligible studies.

Main outcome(s): Exergames induced changes in architectural parameters of individually defined muscles including anatomical cross-sectional area, fascicle length, muscle thickness, pennation angle and physiological cross-sectional area.

Quality assessment / Risk of bias analysis: In the case of qualitative synthesis, the Downs \& Black checklist will be used for quality assessments of both nonrandomised and randomised trials. Additionally, The Cochrane Collaboration's tool for assessing the risk of bias in randomised trials will be used for randomised controlled trials. In case performing a meta-analysis, the GRADE handbook for grading quality of evidence and strength of recommendations will be followed for assessing evidence levels of meta-analyses.

Strategy of data synthesis: Nonrandomised and randomised controlled trials will be included in the qualitative synthesis. In the case of sufficient and homogenous data, randomised controlled trials will be included in meta-analyses. Review Manager (RevMan version 5.4.1) computer program will be used for performing meta-analyses.
Subgroup analysis: Subgroup analysis will be performed in the case of statistically significant heterogeneity and sufficient data in quantitative syntheses based on the type of exergame intervention, study duration, type of muscle architectural parameter or type of individual muscle.

Sensitivity analysis: Sensitivity analysis will be applied across the risk of bias assessment tables in a case of statistically significant heterogeneity in a metaanalysis.

Language: English.

Country(ies) involved: Japan and the United Kingdom.

Keywords: ACSA; Exergame; Fascicle Length; Muscle Architecture; Muscle Thickness; Muscle Volume; PCSA; Pennation Angle; Systematic Review; Video Game.

Contributions of each author:

Author 1 - Nami Shida - The first author will independently screen the citations obtained through database searches, will independently assess the quality and risk of bias of eligible studies, will independently extract data from eligible studies and will contribute to the writing process of the manuscript.

Email: shida@tmu.ac.jp

Author 2 - Gokhan Yagiz - The second author will perform the database searches, will independently screen the citations obtained through database searches, will independently assess the quality and risk of bias of eligible studies, will independently extract data from eligible studies and will contribute to the writing process of the manuscript.

Email: elp8f4@bangor.ac.uk

Author 3 - Takumi Yamada - The last author will involve discussions about selecting eligible studies and data extractions when disagreements arise between the first and second authors. Additionally, the last author will provide feedback on the final manuscript.

Email: yamada@tmu.ac.jp 